\section{Acute infections}

\section{in intravenous}

\section{drug users}

\section{Thomas W Lavender, registrar in infectious diseases; Brendan McCarron, consultant in infectious diseases}

\section{James Cook University Hospital NHS Foundation Trust, Middlesbrough, UK}

Mortality in injecting drug users (IDUs) is up to 22 times higher than for the ageadjusted population. ${ }^{1}$ Despite increased provision of needle and syringe programmes, reduced needle and syringe sharing, and higher uptake of hepatitis B $(\mathrm{HBV})$ vaccination, ${ }^{2}$ IDUs remain vulnerable to a range of infections with potentially life-threatening complications. In this paper, we describe the epidemiology, presentation, diagnosis and management of infections in IDUs who may present to acute physicians and the management of drug dependency on acute medical wards.

\section{HIV and hepatitis B and C}

Almost half of IDUs are positive for hepatitis $\mathrm{C}$ virus (HCV) antibody, and $90 \%$ of newly diagnosed $\mathrm{HCV}$ infections in the UK occur in IDUs. Conversely, $<1 \%$ of IDUs are positive for hepatitis B virus (HBV) surface antigen, accounting for $4.4 \%$ of all reported cases of HBV, which reflects increased uptake of $\mathrm{HBV}$ vaccination in IDUs from $37 \%$ in 2001 to $76 \%$ in 2011. The prevalence of human immunodeficieny virus (HIV) in IDUs in the UK is about $1 \%$ - lower than in many other European countries. ${ }^{2}$ Based on the assumption that all IDUs, particularly those presenting with evidence of immunosuppression or hepatitis, should be screened for blood-borne viruses, we do not discuss these conditions further in this paper.

\section{Skin and soft-tissue infections}

In $2009,28 \%$ of IDUs reported an injection-site infection, ${ }^{3}$ with complications of such infections ranging from uncomplicated cellulitis and localised abscesses to life-threatening necrotising fasciitis and severe sepsis. Staphylococcus aureus and Group A streptococci (GAS) account for most isolated organisms, although Gramnegative and polymicrobial infections frequently occur; $6.9 \%$ of methicillinsensitive $S$ aureus (MSSA) bacteraemia, $3.1 \%$ of methicillin-resistant $S$ aureus (MRSA) bacteraemia and one in five severe GAS infections are associated with IDUs. ${ }^{3-5}$ Subcutaneous or intramuscular injection ('skin popping') is a major risk factor for abscess formation. ${ }^{6}$

In IDUs presenting with skin and softtissue infections (SSTIs), assessment of the site and severity of infection is key. Early ultrasound imaging is useful to confirm the presence of an abscess requiring surgical intervention or an infected iliofemoral venous thrombosis, which is commonly associated with groin infection., ${ }^{7,8}$ Blood cultures, pus swabs (when clinically indicated) and a chest X-ray should be taken for all patients, who should also be assessed thoroughly for infective endocarditis, with a low threshold for performing an echocardiogram. Computed tomography (CT) or magnetic resonance imaging (MRI) may be necessary in cases of suspected pyomyositis or osteomyelitis.

Antibiotic therapy for uncomplicated cellulitis should cover $S$ aureus and streptococci and MRSA should be covered if there is evidence of previous infection or colonisation. First choice therapy is intravenous (IV) flucloxacillin at a dose of $1-2 \mathrm{~g}$ administered every 6 hours; in clinically stable patients with penicillin allergy or difficult intravenous access, oral clindamycin is a useful alternative. For patients with suspected necrotising fasciitis, abscess or pyomyositis, initial therapy should also cover Gram-negative and anaerobic organisms, and urgent surgical input should be sought. ${ }^{9}$ Depending on the extent and complexity of the SSTI, the length of therapy required may range from 10 days to 6 weeks. All patients with bacteraemia caused by $S$ aureus should receive at least 2 weeks of intravenous therapy and undergo an echocardiogram. ${ }^{10}$

\section{Pulmonary infections}

Intravenous drug users have a 10-fold increased risk of community-acquired pneumonia (CAP), including aspiration pneumonia, and a greater incidence of pulmonary tuberculosis compared with the general population. This relates to smoking, alcohol abuse, poor nutrition and homelessness. Organisms commonly causing CAP in this group include Streptococcus pneumoniae, S aureus, Haemophilus influenzae and Klebsiella pneumoniae. ${ }^{11,12}$

Septic pulmonary emboli can seed from injection-site infections and tricuspid valve endocarditis and usually present with high fever and symptoms suggestive of pulmonary emboli. ${ }^{11}$ Lung abscesses result typically from aspiration, frequently due to Kpneumoniae ${ }^{13}$ or septic emboli. Although chest X-ray is sufficient to demonstrate the pulmonary infection in most cases, CT can be useful to confirm cavitation and the distribution of infection and to exclude pulmonary embolus.

Choice of antibiotic therapy is dependent on the organism isolated from blood or sputum and may need to be given for a prolonged period - 4 weeks or more depending on clinical progress. Empirical treatment for lung abscesses should cover $S$ aureus, Gram-negative bacteria and anaerobes.

\section{Infective endocarditis}

The incidence of infective endocarditis (IE) is significantly elevated in IDUs. Injected particulate matter causes endothelial damage, which is followed by infection from high injected bacterial loads associated with relative immune suppression. ${ }^{14}$ Most cases of right-sided IE, particularly tricuspid-valve IE, occur in IDUs. However, right- and left-sided infections occur with equal incidence in IDUs. ${ }^{15}$ Staphylococcus aureus accounts for up to three-quarters of cases, followed by streptococci and, less commonly, Gram-negative bacilli and fungi. ${ }^{16}$ Septic emboli are common in IE caused by $S$ aureus and can involve the lungs (right-sided IE) and the kidney, brain and bone (left-sided IE). ${ }^{16,17}$

The choice and duration of antibiotic therapy are as for non-IDUs with IE. Although there are obvious dangers in discharging IDUs to receive outpatient parenteral antibiotic therapy (OPAT), appropriately selected, counselled and monitored 
patients can be treated safely and successfully and may respond better in the community than during a long inpatient stay. ${ }^{18}$ Short-course treatment of 2-4 weeks can be considered for right-sided IE due to MSSA, ${ }^{19}$ and combination oral therapy can be considered in extreme cases where intravenous access is not possible or the patient is unwilling to stay in hospital; ${ }^{20}$ such cases should be discussed with an infection expert. Surgery for IE is not generally advisable due to the potential for prosthetic valve infection, although some authors support implantation of bioprosthetic valves, with $41 \%$ survival at 10 years reported. ${ }^{21}$

\section{Anthrax}

Prior to 2009, only one case of anthrax had ever been reported in a heroin user worldwide. In 2009-10, 52 confirmed cases were reported; these were predominantly in injecting heroin users in Scotland, who were all thought to have used the same contaminated batch of heroin: $93 \%$ presented with SSTIs and 17 died. ${ }^{3,22}$ No cases were reported in the UK in 2011, although cases re-emerged in 2012, with 12 reported across northern Europe. ${ }^{23}$

Bacillus anthracis causes anthrax when spores, which can survive in the environment for decades, enter the body through abrasions or injection, inhalation or ingestion. Cutaneous disease presents 5-7 days after inoculation as a small pruritic papule, which enlarges, blisters and ulcerates to leave a painless necrotic ulcer with a black eschar. The word anthrax, from the Greek for coal, refers to the typical black eschar, although this was present in only one patient in the 2009-10 outbreak..$^{22,24}$ Extensive oedema in the surrounding tissues, regional lymphadenopathy and lymphangitis develop. ${ }^{24}$ Mortality is $<1 \%$ with antibiotic therapy, typically ciprofloxacin or doxycycline, and $20 \%$ without.

Inhalational anthrax results in haemorrhagic mediastinitis when inhaled spores multiply in mediastinal lymph nodes. ${ }^{25}$ Prodromal symptoms include fever, cough and dyspnoea, which are followed by a rapidly fulminant bacteraemic phase leading to respiratory failure, shock and death within 5 days. Antibiotic therapy and anthrax antiserum can be successful if initiated during the prodromal phase, although the fulminant phase is almost always fatal. ${ }^{26}$ Chest radiography is typically abnormal; widening of the mediastinum secondary to mediastinitis is classic, although pleural effusions, hilar abnormalities, pulmonary infiltrates and consolidation also occur. ${ }^{26}$

\section{Clostridial infections}

Wound botulism occurs when wounds, including injecting sites, are infected with Clostridium botulinum. Prior to 2000, no cases had been reported in the UK, but 163 cases were reported in IDUs from 2000 to 2010, mostly in heroin users injecting into muscle or 'skin popping.',27 Botulinum toxin, one of the most potent toxins known, binds irreversibly to presynaptic membranes in the neuromuscular junction. This blocks acetylcholine release, which typically

\section{Key points}

Intravenous drug users (IDUs) are vulnerable to a wide range of potentially lifethreatening infections, including rare infections such as wound botulism and cutaneous anthrax

All active and previous IDUs should be offered blood-borne virus screening when in contact with healthcare services

In IDUs with skin and soft-tissue infections, thorough assessment of the site and severity is key

Careful assessment for endocarditis, including echocardiography, is required in IDUs presenting with bloodstream infections, septic pulmonary emboli and infected deep venous thromboses

IDUs are at increased risk of pulmonary infection, and septic pulmonary emboli are commonly associated with right-sided endocarditis, infected deep venous thromboses and bloodstream infection

KEY WORDS: Injecting drug users, endocarditis, bacteraemia, anthrax, botulism

results in a descending symmetrical flaccid paralysis, with cranial nerve palsies, dysphagia and dysarthria. Consciousness is maintained and fever typically is absent. Diagnosis is clinical, with confirmation from serological and neurophysiological studies. Treatment includes early administration of antitoxin, wound debridement and antibiotics, as well as supportive care. ${ }^{27}$

An outbreak of Clostridium novyi infection in Scotland in 2000 again resulted from a large batch of contaminated heroin; 60 cases were identified, most injecting heroin extravascularly. Cases were characterised by multi-organ failure with moderate to severe inflammation at or near an injecting site and marked leucocytosis (median white cell count $60 \times 10^{9} / 1$ ). Mortality was $87 \%$ in confirmed cases. ${ }^{3,28}$

Only two cases of tetanus were reported in IDUs in England and Wales between 1984 and 2000. However, an outbreak of 25 cases occurred among IDUs in the UK between 2003 and 2004. Eight cases were reported in 2005-2009 and one in 2010. ${ }^{3}$

\section{Managing IDUs on medical wards}

Intravenous drug users can be challenging patients to manage on medical wards, with aggressive behaviour, illicit drug use while in hospital and early self-discharge commonly encountered. Good management requires a frank and non-judgemental approach, and clear behavioural boundaries should be outlined, including explanations of the consequences of abusing staff, demanding medications other than those prescribed and obstructing the continuity of care. ${ }^{29}$ Opiates and other drugs that could be misused, including intravenous cyclizine, ${ }^{30}$ should be prescribed by a single sufficiently experienced prescriber.

Identification of IDUs is crucial - both to determine clinical risk and to minimise the risk of unexpected withdrawal. An accurate drug history, including route of administration, should be completed for all patients on admission, acknowledging that this may need to be repeated until an accurate picture is gained. All IDUs should be asked about the use of other substances, especially alcohol and benzodiazepines. For patients on opioid maintenance therapy, the dose and timing of the last dose should 
be confirmed with the dispensing centre at the first opportunity.

Although requests for analgesia are often interpreted by healthcare staff as drug-seeking behaviour, withdrawal symptoms and pain must be adequately treated. If evidence indicates opiate withdrawal, 10-20 mg of methadone can be given safely until the regular prescribed dose is confirmed. For acute pain management, non-opioid analgesia, including paracetamol and non-steroidal anti-inflammatory drugs, should be optimised, with short-acting opioids added if required. IDUs typically require higher than usual doses of opioid analgesics, which should subsequently be converted to regularly dosed slow-release preparations while long-acting methadone is continued to relieve symptoms of withdrawal. ${ }^{29}$ When considering the use of adjunctive rifampicin in infections caused by $S$ aureus, physicians should be aware that the dose of methadone may need to be increased; this requires close monitoring to avoid methadone withdrawal. ${ }^{31}$

\section{Conclusions}

Although infections in IDUs can be challenging to manage, they can be satisfying to look after with the right approach. Skin and soft-tissue infections and complications of bacteraemia remain common, but outbreaks of rare infections do occur, so it is essential to remain alert for severe or unusual presentations.

\section{References}

1 Frischer M, Goldberg D, Rahman M, Berney L. Mortality and survival among a cohort of drug injectors in Glasgow, 1982 1994. Addiction 1997;92:419-27.

2 Health Protection Agency, Health Protection Scotland, Public Health Wales, CDSC Northern Ireland, and the CRDHB. Shooting up: infections among people who inject drugs in the UK 2009. London: Health Protection Agency, 2010. www.hpa. org.uk/webc/HPAwebFile/HPAweb_C/ 1287143384395 [Accessed 29 August 2013].

3 Health Protection Agency, Health Protection Scotland, Public Health Wales, and Public Health Agency Northern Ireland. Shooting up: infections among people who inject drugs in the UK 2010. London: Health Protection Agency, 2011. www.hpa.org.uk/ webc/HPAwebFile/HPAweb_C/1317131377664 [Accessed 29 August 2013].
4 Ebright JR, Pieper B. Skin and soft tissue infections in injection drug users. Infect Dis Clin North Am 2002;16:697-712.

5 Lamagni TL, Neal S, Keshishian C et al. Severe Streptococcus pyogenes infections, United Kingdom, 2003-2004. Emerg Infect Dis 2008;14:201-9.

6 Murphy EL, DeVita D, Liu H et al. Risk factors for skin and soft-tissue abscesses among injection drug users: a case-control study. Clin Infect Dis 2001;33:35-40.

7 Roberts S, Chambers S. Diagnosis and management of Staphylococcus aureus infections of the skin and soft tissue. Intern Med J 2005;35:S97-105.

8 Mackenzie AR, Laing RB, Douglas JG et al. High prevalence of iliofemoral venous thrombosis with severe groin infection among injecting drug users in North East Scotland: successful use of low molecular weight heparin with antibiotics. Postgrad Med J 2000;76:561-5.

9 Anaya DA, Dellinger EP. Necrotizing softtissue infection: diagnosis and management. Clin Infect Dis 2007;44:705-10.

10 Thwaites GE, Edgeworth JD, GkraniaKlotsas E et al. Clinical management of Staphylococcus aureus bacteraemia. Lancet Infect Dis 2011;11:208-22.

11 Hind CR. Pulmonary complications of intravenous drug misuse. 2. Infective and HIV related complications. Thorax 1990;45:957-61.

12 Deiss RG, Rodwell TC, Garfein RS. Tuberculosis and illicit drug use: review and update. Clin Infect Dis 2009;48:72-82.

13 Wang JL, Chen KY, Fang CT et al. Changing bacteriology of adult community-acquired lung abscess in Taiwan: Klebsiella pneumoniae versus anaerobes. Clin Infect Dis 2005;40:915-22.

14 Frontera JA, Gradon JD. Right-side endocarditis in injection drug users: review of proposed mechanisms of pathogenesis. Clin Infect Dis 2000;30:374-9.

15 Beynon RP, Bahl VK, Prendergast BD. Infective endocarditis. $B M J$ 2006;333:334-9.

16 Wilson LE, Thomas DL, Astemborski J et al. Prospective study of infective endocarditis among injection drug users. J Infect Dis 2002;185:1761-6.

17 Ruotsalainen E, Sammalkorpi K, Laine J et al. Clinical manifestations and outcome in Staphylococcus aureus endocarditis among injection drug users and nonaddicts: a prospective study of 74 patients. BMC Infect Dis 2006;6:137.

18 Ho J, Archuleta S, Sulaiman Z, Fisher D. Safe and successful treatment of intravenous drug users with a peripherally inserted central catheter in an outpatient parenteral antibiotic treatment service. J Antimicrob Chemother 2010;65:2641-4.
19 Yung D, Kottachchi D, Neupane B et al. Antimicrobials for right-sided endocarditis in intravenous drug users: a systematic review. J Antimicrob Chemother 2007;60:921-8.

20 Murray RJ. Staphylococcus aureus infective endocarditis: diagnosis and management guidelines. Intern Med J 2005;35:S25-44.

21 Rabkin DG, Mokadam NA, Miller DW et al. Long-term outcome for the surgical treatment of infective endocarditis with a focus on intravenous drug users. Ann Thorac Surg 2012;93:51-7.

22 Health Protection Scotland. An outbreak of anthrax among drug users in Scotland, December 2009 to December 2010. A report on behalf of the National Anthrax Outbreak Control Team. Glasgow: Health Protection Scotland, 2011. www.documents.hps.scot. nhs.uk/giz/anthrax-outbreak/anthraxoutbreak-report-2011-12.pdf [Accessed 5 August 2013].

23 Health Protection Report. Anthrax cases among drug users in Europe: third update. Health Protection Report 2012;6:2. www.hpa. org.uk/hpr/archives/2012/news4412. htm\#anthrax1211 [Accessed 29 August 2013].

24 Dixon TC, Meselson M, Guillemin J, Hanna PC. Anthrax. N Engl J Med 1999;341:815-26.

25 Abramova FA, Grinberg LM, Yampolskaya OV, Walker DH. Pathology of inhalational anthrax in 42 cases from the Sverdlovsk outbreak of 1979. Proc Natl Acad Sci USA 1993;90:2291.

26 Holty JE, Bravata DM, Liu $\mathrm{H}$ et al. Systematic review: a century of inhalational anthrax cases from 1900 to 2005. Ann Intern Med 2006;144:270-80.

27 Brett MM, Hallas G, Mpamugo O. Wound botulism in the UK and Ireland. J Med Microbiol 2004;53:555-61.

28 McGuigan CC, Penrice GM, Gruer L et al. Lethal outbreak of infection with Clostridium novyi type A and other sporeforming organisms in Scottish injecting drug users. J Med Microbiol 2002;51:971-7.

29 Haber PS, Demirkol A, Lange K, Murnion B. Management of injecting drug users admitted to hospital. Lancet 2009;374:1284-93.

30 Ruben SM, McLean PC, Melville J. Cyclizine abuse among a group of opiate dependents receiving methadone. Br J Addict 1989;84:929-34.

31 Kreek MJ, Garfield JW, Gutjahr CL, Giusti LM. Rifampin-induced methadone withdrawal. N Engl J Med 1976;294:1104-6.

\section{Address for correspondence:}

Dr TW Lavender, James Cook

University Hospital NHS Foundation

Trust, Marton Road, Middlesbrough

TS4 3BW.

Email: thomas.lavender@nhs.net 